\title{
Análisis de resultados de las pruebas nacionales de bachillerato de educación media de los colegios que conforman las direcciones regionales de educación en zonas fronterizas costarricenses. 2013-2016
}

High School Performance in the National Baccalaureate Tests in Regional Education Jurisdictions in Costa Rican Border Areas, 2013-2016

\section{Desempenho dos colégios nos exames nacionais de bacharelado nas Direcções Regionais de Educação nas zonas fronteiriças da Costa Rica, 2013-2016}

\author{
Cindy Vanessa Artavia-Aguilar \\ Universidad Nacional \\ Centro de Investigación y Docencia en Educación \\ Heredia, Costa Rica \\ cindy.artavia.aguilar@una.cr \\ D https://orcid.org/0000-0002-2179-6808 \\ Guillermo Calderón-Ramírez \\ Universidad Nacional \\ Centro de Investigación y Docencia en Educación \\ Heredia, Costa Rica \\ gcalderon@una.cr \\ Andrea Ramírez-González \\ Centro de Investigación y Docencia en Educación \\ Heredia, Costa Rica \\ andrea.ramirez.gonzalez@una.cr \\ https://orcid.org/0000-0002-9437-0867 \\ Francisco González-Alvarado \\ Universidad Nacional \\ Centro de Investigación y Docencia en Educación \\ Heredia, Costa Rica \\ francisco.gonzalez.alvarado@una.cr \\ https://orcid.org/0000-0001-8561-0411 \\ Pablo Chaverri-Chaves \\ Universidad Nacional \\ Centro de Investigación y Docencia en Educación \\ Heredia, Costa Rica \\ pablochaverri@yahoo.com \\ (D) https://orcid.org/0000-0002-2639-4242 \\ Eddy Cruz-Arroyo \\ Universidad Nacional \\ Centro de Investigación y Docencia en Educación \\ Heredia, Costa Rica \\ ecruz@una.cr \\ D https://orcid.org/0000-0003-1741-1677
}

Recibido • Received • Recebido: 28 / 08 / 2018

Corregido • Revised • Revisado: 06 / 12 / 2018

Aceptado • Accepted • Aprovado: 02/ 05 / 2019 
doi: http://dx.doi.org/10.15359/ree.23-3.1

URL: http://www.una.ac.cr/educare

CORREO: educare@una.cr

Resumen: En el marco del programa Perfiles, dinámicas y desafíos de la educación costarricense, del Centro de Investigación y Docencia en Educación de la Universidad Nacional, este artículo abre un espacio de diálogo y reflexión nacional acerca de las pruebas nacionales de bachillerato en educación media en Costa Rica y el desempeño de los colegios en las zonas fronterizas. Este trabajo se plantea como objetivo analizar los resultados de las Pruebas Nacionales de Bachillerato en Educación Media (PNB-EM), en las zonas fronterizas del país, con la intención de visualizar los avances en materia educativa y sus repercusiones a nivel social. El análisis de este documento surge de los resultados de las PNB-EM reportados por el MEP en las 8 direcciones regionales de educación (DRE) ubicadas en áreas fronterizas norte y sur de nuestro país, y la relación de estos resultados con el índice de desarrollo social (IDS) reportado por los cantones que componen dichas DRE. Los datos estadísticos con los que se trabajó fueron de dos ámbitos, el educativo en relación con las PNB-EM en lo que respecta a la promoción, nota promedio obtenida y el índice de desarrollo social cantonal del año 2013. El proceso de análisis de los datos refleja algunas conclusiones importantes en materia de equidad, inclusión educativa y condiciones de desarrollo social a tomar en consideración para la interpretación de los resultados de las PNB-EM en el país.

Palabras claves: Pruebas nacionales de bachillerato; educación media; zonas fronterizas; índice de desarrollo social.

Abstract: In the frame of the Program Perfiles, Dinámicas y Desafíos de la Educación Costarricense, from the Centro de Investigación y Docencia en Educación (CIDE), of the National University of Costa Rica, this article aims to open a space of national dialog and reflection about the standardized tests of the national baccalaureat (pruebas nacionales de bachillerato or PNB) for secondary education in Costa Rica, and the performance of secondary schools in border zones. Its specific goal is to analyze the results of these national tests of secondary education in Costa Rica as instruments to certify knowledge in the border zones of the country, with the intention to visualize advancements in education and their social repercussions. The analysis is based on the results of the PNB reported to the Education Ministry by the eight regional education authorities located in border zones from the north and south of the country, and their relation with the Social Development Index (Índice de Desarrollo Social or IDS) of the cantons under these authorities jurisdiction. The statistical data were worked in two dimensions, one related to the PNB results in terms of general promotion, and average grades; and the second one related to the IDS for 2013. The data analysis process let us show some transcendental conclusions regarding equity, educative inclusion, and social development conditions to take into consideration when interpreting PNB results in the country.

Keywords: National secondary education stardadized Tests; secondary education; border zones, social development index.

Resumo: Este artigo, no âmbito do programa Perfis, Dinâmicas e Desafios da Educação da Costa Rica, do Centro de Investigação e Ensino em Educação da Universidade Nacional, pretende abrir um espaço de diálogo e reflexão nacional sobre os testes de bacharelado do ensino médio nacional na Costa Rica e o desempenho das escolas nas áreas de fronteira. $O$ objetivo deste artigo é analisar os resultados dos Testes de Bacharelado em Ensino Médio Nacional (PNB-EM) como um instrumento de certificação de conhecimento, nas áreas de fronteira do país, com a intenção de visualizar os 


\begin{abstract}
avanços da educação e suas repercussões em nível social. A análise deste documento surge dos resultados do PNB-EM relatados pelo MEP nas 8 direcções regionais de educação (DRE) localizadas nas áreas fronteiriças norte e sul do nosso país, e a relação destes resultados com os Indices de Desenvolvimento Social (IDS) relatado pelas regiões que compõem os DRE. Os dados estatísticos que foram trabalhados partiram de duas áreas, a educacional em relação ao PNB-EM em termos de promoção, as notas obtida e o índice de desenvolvimento social cantonal para o ano de 2013. O processo de análise de dados reflete algumas conclusões transcendentais em termos de equidade, inclusão educacional e condições de desenvolvimento social a serem levadas em consideração para a interpretação dos resultados do PNB-EM no país.
\end{abstract}

Palavras-chave: Testes nacionais do ensino médio; educação média; áreas de fronteira; índice de desenvolvimento social.

\title{
Introducción
}

El presente artículo es el resultado del esfuerzo de un proceso de investigación que se realiza en el Centro de Investigación y Docencia en Educación (CIDE) de la Universidad Nacional; se circunscribe en el marco del proyecto de investigación "Balance crítico de objetivos, modelo evaluativo, resultados y utilidad de las Pruebas Nacionales de Bachillerato en Educación Media (PNB-EM) como instrumento de certificación del conocimiento de estudiantes 2012-2016", y tiene como objetivo analizar las pruebas nacionales de bachillerato como instrumento de certificación del conocimiento, a partir de un estudio de carácter diagnóstico sobre los objetivos, modelo evaluativo, resultados y utilidad, que contribuya al debate sobre esta política educativa. Dicho estudio es un balance de tres décadas de aplicación de las pruebas nacionales de bachillerato y los resultados obtenidos en ese lapso de tiempo, lo que permite visualizar los avances en materia educativa y sus repercusiones a nivel social.

En el presente artículo, se consigna un estudio particularizado en relación con los resultados de las PNB-EM en las Direcciones Regionales de Educación (DRE) ubicadas en zonas fronterizas norte y sur del país, en el periodo 2013 -2016, y su relación con el índice de desarrollo social (IDS) publicado en el año 2013, que nos permita discutir realidades socioeducativas en esos contextos y, de esta manera, contribuir con información sustantiva y pertinente que oriente la discusión nacional sobre los resultados y los posibles efectos de estas pruebas en estos espacios territoriales del país.

\section{Antecedentes}

La Universidad Nacional (UNA), comprometida con los procesos de desarrollo nacional y grandes temas que responden a una realidad acorde a las necesidades de la ciudadanía, ha venido ofreciendo respuestas en el campo de la educación desde el CIDE, desde hace más de cuatro décadas. En este contexto, el programa de investigación "Perfiles, dinámicas y desafíos de la educación costarricense", iniciativa académica del Centro, que integra un grupo de 
doi: http://dx.doi.org/10.15359/ree.23-3.1

URL: http://www.una.ac.cr/educare

CORREO: educare@una.cr

trabajo interdisciplinario, pretende contribuir al debate nacional sobre las pruebas nacionales de bachillerato en educación media (PNB-EM), especialmente por la evidencia de diferencias profundas en los resultados obtenidos por el estudiantado. Asimismo, el tema ha despertado el interés de diferentes sectores sociales, políticos y medios de comunicación, así como al interior del Ministerio de Educación Pública (MEP).

Existe documentación valiosa en el Ministerio de Educación Pública relacionada con las pruebas nacionales de bachillerato; destacan los Boletines de Supervisión Nacional, que desde el año 1984 hasta el año 2000 publicaron directrices, acuerdos, propuestas curriculares, e información relacionada con estas.

Otras fuentes secundarias hacen mención directa a las PNB-EM, el rendimiento y evaluaciones anuales de los resultados en todo el país. Cabe destacar, investigaciones del MEP, trabajos finales de graduación de la Universidad Nacional y Universidad de Costa Rica, investigaciones a nivel latinoamericano y otros documentos. También, se cuenta con informes anuales que incluyen datos estadísticos a nivel nacional, por regiones educativas, colegios y estudiantes, bases de datos cuantitativos y variables educativas.

Por lo anterior, cabe destacar el rol que ha asumido el Consejo Superior de Educación (CSE) como ente superior en materia de política educativa nacional, el cual ha realizado distintas discusiones que se evidencian en las actas del Consejo. De esta manera, como parte del análisis de estas mediciones, el CSE manifestó el interés de someter estas pruebas a evaluación externa, la cual se concretó en la investigación presentada por la Universidad de Costa Rica en el año 2006 denominada Evaluación externa de las pruebas nacionales de bachillerato de la educación media, la cual fue realizada por un equipo de investigación liderado por el Dr. Juan Manuel Esquivel Alfaro, con la finalidad de "realizar una evaluación formativa y externa de las pruebas nacionales de bachillerato de la educación formal" (Esquivel et al., 2006, p. 9). Esta investigación enfatizó "como recomendación fundamental la necesidad de mantener y fortalecer las pruebas nacionales de bachillerato en las tres dimensiones generales objeto de esta evaluación: lo político-educativo, lo técnico-metodológico y lo técnico-administrativo" (Esquivel, et al., 2006, p. 59).

A partir de la evaluación externa de las PNB-EM en el 2006, la Defensoría de los Habitantes de la República de Costa Rica ha emitido diversas consideraciones, informes y recomendaciones técnicos-legales al CSE en relación con los objetivos, modelo evaluativo y estructura técnica de las pruebas nacionales de bachillerato del MEP.

Asimismo, con base en estudios publicados por el programa del Estado de la Educación e informes técnicos producidos por el MEP, es posible documentar una brecha en los resultados de las PNB-EM que agudiza diferencias sustantivas respecto a las modalidades, los sectores y las regiones educativas (Programa Estado de la Nación, 2013). En ese sentido, cabe mencionar que la poca investigación localizada en relación con las PNB-EM no establece ningún tipo de vinculación con indicadores sociales o de otra índole; tampoco se establecen criterios de 
comparación o análisis entre regiones educativas y tipo de institución. Los informes sobre evaluaciones de las pruebas nacionales son anuales y no presentan resultados en una línea de tiempo superior que permitan establecer comparaciones por períodos, tal como lo realiza la investigación marco de este artículo que retoma un balance de tres décadas de las PNB-EM.

Una limitación de los estudios realizados radica en que no profundizan en los resultados de las pruebas nacionales de bachillerato en las regiones educativas fronterizas ni en la población en desventaja social, migrantes, zonas costeras u otros grupos de vulnerabilidad social; tampoco se contextualizan los resultados de las PNB-EM a las regiones de procedencia del estudiantado. De ahí radica la importancia de analizar, de forma particular, los resultados de las pruebas nacionales de bachillerato en las zonas fronterizas y relacionar los resultados con indicadores sociales (Ministerio de Planificación Nacional y Política Económica, 2013) que nos permitan discutir realidades socioeducativas de las regiones educativas fronterizas.

\section{Marco teórico o referentes conceptuales}

\section{Contexto normativo de las pruebas nacionales de bachillerato en educación media}

El Reglamento de valuación de los aprendizajes (REA) vigente fue aprobado por el Consejo Superior de Educación de la República de Costa Rica, mediante acuerdo N. ${ }^{\circ}$ 06-72-2017 de la sesión ordinaria N. ${ }^{\circ}$ 72-2017 celebrada el 18 de diciembre de 2017 y el Poder Ejecutivo lo ejecuta para su respectiva publicación en el Diario oficial La Gaceta del día martes 6 de febrero de 2018 según decreto N. ${ }^{\circ}$ 40862-MEP (MEP, 2018). El REA establece las disposiciones normativas que regulan el proceso de evaluación de los aprendizajes y la implementación del sistema de convivencia estudiantil en el sistema educativo costarricense.

El REA (MEP, 2018), en el capítulo V denominado Pruebas Nacionales, expone las normas que regulan las dispocisiones generales, la gestión administrativa, las pruebas nacionales de bachillerato en educación media, las acciones fraudulentas, la comunicación nacional y aplicación de los resultados y las disposiciones finales sobre las pruebas nacionales.

De acuerdo con el interés de este estudio, los artículos 79, 80 y 85 de este reglamento (MEP, 2018) indican que la finalidad de las pruebas nacionales de bachillerato en educación media (PNB-EM) es de carácter censal y de certificación, lo cual implica que toda persona estudiante que curse el último año de educación diversificada o el tercer nivel del sistema modular de los institutos de educación comunitaria (IPEC), centro integrado de educación de personas adultas (CINDEA) y colegio nacional de educación a distancia (CONED) y que desee culminar la educación diversificada debe aprobar estas pruebas con el fin de obtener el título de Bachiller en Educación Media y, con ello, alcanzar el perfil académico establecido en el currículo de este nivel del sistema educativo. 
doi: http://dx.doi.org/10.15359/ree.23-3.1

URL: http://www.una.ac.cr/educare

CORREO: educare@una.cr

Tal como lo establece el artículo 96 del REA, las PNB-EM son pruebas comprensivas de los contenidos de los programas de estudio de la educación diversificada, aprobados por el Consejo Superior de Educación, para cada una de las asignaturas: Español, Estudios Sociales, Educación Cívica, Ciencias, Matemáticas y lengua extranjera. En la asignatura de Ciencias, cada estudiante escogerá entre Física, Química o Biología. En lengua extranjera, la persona estudiante escogerá entre Inglés o Francés.

El artículo 102 (MEP, 2018) determina los elementos que intervienen en la fórmula para el cómputo de la calificación final de la PNB-EM, la cual se calcula mediante la combinación porcentual de la obtenida en la respectiva prueba de bachillerato que representa un $60 \%$ con la nota de presentación de cada estudiante que representa un $40 \%$. Para ello, se establecen las asignaturas que se incluyen en el cálculo de la nota de presentación para todas las modalidades de educación diversificada. Al respecto el REA indica que:

La nota de presentación se define como el promedio de las calificaciones obtenidas por la persona estudiante en décimo año y en los dos primeros trimestres de undécimo año en Español, Matemática, Estudios Sociales y Educación Cívica, Inglés o Francés (según corresponda) y Biología, Química o Física (según corresponda). Para el caso de los colegios técnicos se considerarán las calificaciones obtenidas por la persona estudiante en décimo año, undécimo año y los dos primeros trimestres de duodécimo año en las mismas asignaturas señaladas anteriormente.

Para los estudiantes del III Nivel del Plan de Estudios de Educación de Adultos, se tomarán en cuenta las calificaciones de las asignaturas antes citadas en los periodos aprobados en cada módulo al momento de enviar la información respectiva. (MEP, 2018, p. 85)

Asimismo, en el artículo 102 (MEP, 2018) se indica que el valor porcentual que representa la nota de cada examen por asignatura es de $60 \%$ de la calificación final de cada prueba de bachillerato.

Al mismo tiempo, el artículo 102 señala la forma de obtener la calificación final de la prueba final de bachillerato en español que "será el promedio de las calificaciones que obtenga el postulante en Composición y Ortografía y Gramática y Literatura" (MEP, 2018, p. 86).

La aprobación de la PNB-EM, para cada estudiante respecto a la asignatura objeto de medición, debe lograr una calificación final igual o superior a 70, en una escala de 1 a 100, tal como lo indican los artículos 102 y 105 del REA (MEP, 2018).

Los artículos 82, 88 y 93 del reglamento en cuestión mencionan que la instancia del Ministerio de Educación Pública es responsable de planificary dirigir los procesos de elaboración, administración, calificación y comunicación de los resultados de las PNB-EM, es la Dirección de Gestión y Evaluación de la Calidad (DGEC) (MEP, 2018). Para ello, la DGEC cuenta con personal asesor en colaboración de las asesorías nacionales y regionales de las asignaturas objeto de medición, así como con la participación de personal docente y profesional especializado (MEP, 
2018). De esta manera, con el liderazgo de esta dirección y en conjunto con las instituciones de educación secundaria, es posible llevar a cabo, en todo el territorio nacional y en un misma calendarización, la realización de las PNB-EM.

\section{Las evaluaciones estandarizadas como mecanismo de certificación de los aprendizajes}

En el modelo evaluativo que sustenta el diseño y ejecución de las Pruebas Nacionales de Bachillerato constituye una tarea de suma complejidad definir conceptualmente la evaluación; entre otras razones, por la existencia de diferentes planteamientos teóricos y modelos explicativos que le otorgan una evolución conceptual particular, la cual, de manera mayormente significativa, es promovida por diferentes escuelas de psicología educativa norteamericanas, entre las que resaltan los estudios de Tyler, Suchman, Cronbach, Stuffleam, Stake y House, sobre todo durante el siglo pasado, y quienes le otorgan una preeminencia sustantiva en el marco general de la educación. De igual forma, por la aceptación del reconocimiento de multiplicidad de factores y procesos inherentes al acto mismo de la evaluación.

La evaluación como proceso dinámico, abierto y contextualizado que se desarrolla a lo largo de un periodo de tiempo; ... no es una acción puntual aislada [por lo que se] han de cumplir varios pasos sucesivos durante dicho proceso, para que se puedan dar las tres características esenciales e irrenunciables de toda evaluación: ... obtener información, ... formular juicios de valor [y] tomar decisiones. (Castillo, 2002, p. 25)

En el contexto de la evaluación de los aprendizajes, se asumen repercusiones en el ámbito de lo social que involucran directamente a las familias, los centros educativos y los estudiantes en particular. El MEP la describe en el artículo 3 del REA, como: "un proceso continuo de recopilación de información cualitativa y cuantitativa, que fundamenta la emisión de juicios de valor y la toma de decisiones por parte de la persona docente y el estudiantado, para la mejora progresiva de los procesos de enseñanza y aprendizaje" (MEP, 2018, pp. 23-24).

Las definiciones citadas permiten acercarse a la caracterización del concepto de evaluación promovido por el MEP, reconociendo tres rasgos centrales: el primero es el carácter procesual y centrado en el papel protagónico del personal docente como responsable de la planificación y ejecución de este mismo; el segundo es su variedad instrumental, es decir; la posibilidad de realizarse por medio de diferentes medios, tanto cualitativos, como cualitativos, y por último, su sentido y finalidad práctica, orientada hacia verificar el impacto individual y colectivo de los aprendizajes promovidos, así como emplazar las decisiones en función de los resultados obtenidos. Tales constructos han constituido, en el tiempo, los marcos referenciales para el diseño metodológico, la construcción formal de instrumentos y la aplicación de las PNB-EM, empleadas para la certificación de los aprendizajes y la promoción de estudiantes al finalizar la enseñanza media por casi tres décadas. 
doi: http://dx.doi.org/10.15359/ree.23-3.1

URL: http://www.una.ac.cr/educare

CORREO: educare@una.cr

\section{La regionalización educativa en el Ministerio de Educación Pública}

De acuerdo con Calderón (2015), en Costa Rica el análisis socioeconómico se desarrolla con la creación de las regiones el 15 de febrero de 1985; se divide el país en seis regiones con el objetivo de simplificar la investigación y planificación: Central, Chorotega, Pacífico Central, Brunca, Huetar Atlántica, Huetar Norte.

EI MIDEPLAN (2013) define el tema de desarrollo social como un proceso en el que se pretende que la población mejore su calidad de vida, por lo cual considera la igualdad, participación e inclusión, y reduce las desigualdades sociales en un espacio geográfico. MIDEPLAN (2013) explica la situación social desde una perspectiva territorial con el IDS que tiene en cuenta, para su cálculo, los componentes de educación, economía, salud y participación.

En esta misma línea, el Estado es el actor clave en garantizar mejor calidad de vida de las personas a través de programas y políticas públicas, esto dentro de un territorio que, a pesar de estar dentro de una misma Nación, contiene contrastes que generan su estudio independiente, integrando variables según su sentido de análisis.

La regionalización de la educación, según Barrantes (1997), comienza en el año 1978, pretende mejorar el nivel de calidad de la educación costarricense, teniendo como punto de partida la desconcentración de labores en la administración. Para ello, el MEP conforma veintisiete direcciones regionales de educación que permiten la organización, el reconocimiento de la educación como derecho y el resguardo de su calidad.

Las DRE son instancias administrativas que llevan a cabo la organización, la prestación de servicios de educación y facilitan la atención de las comunidades educativas.

Para el caso que nos ocupa en este estudio, es importante tomar en cuenta el IDS para analizar los resultados de las PNB-EM en las DRE pertenecientes a regiones fronterizas norte y sur del país, comprendidas como aquellos espacios que están determinadas por dinámicas sociales, que considera los límites internacionales que hacen de estas áreas un flujo de intercambio y comercio, como lo señala Meza (2008), autor que apunta lo siguiente:

Son muy pocos y muy parciales, los esfuerzos por entender los Espacios Regionales Fronterizos como partes integrantes de un todo orgánico, cuya especificidad radica en la presencia de límites internacionales que, ... se levantan como testimonios de integración más que de separación. (Meza, 2008, p. 21)

De los países latinoamericanos, gran parte de estos tienen la características de que sus fronteras son marginadas, con altos niveles de pobreza. Esa marginalidad Meza (2008) la"... asocia al grado de articulación o desarticulación respecto a los circuitos nacionales de la economía o, ... a los ejes o corredores viales/económicos que vienen convirtiéndose en el referente para

8

Cindy Vanessa Artavia-Aguilar, Guillermo Calderón-Ramírez, Andrea Ramírez-González, Francisco González-Alvarado, Pablo Chaverri-Chaves y Eddy Cruz-Arroyo

Los artículos de la Revista Electrónica Educare del Centro de Investigación y Docencia en Educación de la Universidad Nacional, Costa Rica, se comparten bajo términos de la Licencia Creative Commons: Reconocimiento, No Comercial, Sin Obra Derivada 3.0 Costa Rica. Las autorizaciones adicionales a las aquí delimitadas se pueden obtener en el correo: educare@una.cr 
estrategias de desarrollo regional" (p. 21). Hay que reconocer que en estos territorios no se tenía la concepción de que fueran espacios en desarrollo y eran vistos únicamente como áreas en las que un país pudiera estratégicamente defenderse y mantener su soberanía (Meza, 2008), se consideran estos espacios valiosos en el crecimiento económico de una Nación.

\section{Equidad y exclusión en educación}

¿Caras de una misma moneda? En una sociedad cada vez más cambiante, con un alto nivel de complejidad y en una multiplicidad de realidades sociales, los sistemas educativos no son ajenos a estos escenarios y están inmersos en realidades diversas a nivel personal, social, cultural y curricular.

Por otra parte, la equidad educativa busca establecer un criterio para identificar las desigualdades sociales y educativas respecto a un horizonte de igualdad de condiciones para todas las personas. El principio de equidad surge desde el reconocimiento de las diversidades personales, sociales, culturales, políticas y de todos los escenarios en los que se desenvuelven los sujetos; es un reconocimiento indiscutiblemente ético de las dimensiones en torno al ser humano y su desarrollo en educación. Como plantea López (2005), "la noción de equidad no compite ni desplaza a la de igualdad, sino que, por el contrario, la integra, [ampliándose] en sus múltiples dimensiones. No hay equidad sin igualdad, sin esa igualdad estructurante que define el horizonte de todas las acciones" (p. 68).

Con el afán de ampliar la visión del tema de equidad en educación, su respectivo análisis y valoración en procesos educativos cada vez más equitativos, se destaca, en la teoría, al menos cuatro principios de equidad relacionados con el acceso, la igualdad de condiciones, estilos y medios de aprendizaje e igualdad de las condiciones sociales y culturales que ayudan a dilucidar cualquier escenario educativo planteado desde la equidad y las igualdades fundamentales (Demeuse, 2004, citado por Pérez, 2015).

En el contexto educativo costarricense y a la luz de los resultados de las pruebas nacionales de bachillerato, el análisis desde estos cuatro principios es imperante para poder valorar las condiciones reales de equidad educativa, o bien, considerar aquellos procesos que conllevan a la desigualdad de las condiciones educativas del estudiantado, especialmente, como compete en este análisis, a la población estudiantil de zonas fronterizas.

Las desigualdades que pueden existir en los sistemas educativos, y los resultados que de estas derivan, pueden reforzar las diferencias sociales, al ofrecer condiciones educativas mínimas y extender las brechas sociales, con el agravante de llevar esta desigualdad a mecanismos estructurados e institucionalizados que son excluyentes, intolerantes e ilegítimos. Según Stubbs (2008), son muchos los grupos que pueden estar en riesgo de exclusión: niñas y mujeres, estudiantes que viven en ámbitos rurales, estudiantes con enfermedades crónicas, discapacidad, en condición de pobreza extrema, prostitución, etc. 
doi: http://dx.doi.org/10.15359/ree.23-3.1

URL: http://www.una.ac.cr/educare

CORREO: educare@una.cr

Ante lo expuesto, se plantea:

Las múltiples formas en que la exclusión se manifiesta en distintos momentos tienen conjuntamente un efecto acumulativo que puede alimentar la exclusión social. Por ello, además de determinar quién es objeto de exclusión, es fundamental descubrir cuándo se produce la exclusión para poder dar una respuesta adecuada y eficaz. (Unesco, 2012, p. 8)

Es importante discernir las formas en las que se presenta la exclusión en educación para poder dar respuesta oportuna y adecuada a situaciones que generan dicha desigualdad; respuestas destinadas a romper barreras de la exclusión, y que promuevan espacios educativos que contemplen todas las realidades estudiantiles.

\section{Marco metodológico}

\section{Enfoque metodológico}

Como parte de la investigación denominada Balance crítico de objetivos, modelo evaluativo, resultados y utilidad de las Pruebas Nacionales de Bachillerato en Educación Media (PNB-EM) como instrumento de certificación del conocimiento de estudiantes 2012-2016, el presente estudio se circunscribe en el propósito relativo a los "resultados" de estas pruebas, particularmente, en el análisis de los resultados de las PNB-EM reportados por el MEP en el período 2013-2016 y obtenidos por el estudiantado de ocho direcciones regionales de educación (DRE) ubicadas en áreas fronterizas norte y sur de nuestro país, y la relación de estos resultados con el IDS reportado por los cantones que componen dichas DRE. En la Tabla 1 se observan las DRE del estudio, según su ubicación respecto a las fronteras de Costa Rica.

Tabla 1: DRE Fronterizas

\begin{tabular}{ll}
\hline Frontera norte de Costa Rica & Frontera sur de Costa Rica \\
\hline - DRE Liberia & - DRE Coto \\
- DRE Zona Norte-Norte & - DRE Grande de Térraba \\
- DRE San Carlos & - DRE Sulá \\
- DRE Sarapiquí & \\
- DRE Guápiles & - \\
\hline
\end{tabular}

Nota: Elaboración propia. 
Es importante aclarar que, aunque la DRE Grande de Térraba no es una región fronteriza propiamente, los motivos de incluirla se deben a que tiene un contacto directo con las DRE de Coto y Sulá como puente de conexión entre estas y, por ende, es una región que forma parte del contexto socioeconómico y las dinámicas territoriales que se dan en la frontera sur costarricense. Otra razón está relacionada a que comparte indicadores del índice de desarrollo social similares a los de Coto y Sulá, todo lo anterior aunado a que la DRE Grande de Térraba tiene uno de sus cantones a pocos kilómetros de la línea fronteriza.

El estudio se basó en un diseño metodológico transversal descriptivo desde un enfoque cuantitativo, con la finalidad de indagar la incidencia y los valores que se manifiestan en una o más variables (Hernández, Fernández y Baptista, 2014), particularmente, los resultados de las PNB-EM en zonas fronterizas y su vinculación con el índice de desarrollo social. Para ello, se describieron y se analizaron algunos indicadores educativos tales como porcentaje de aprobación en las PNB-EM y el promedio de la nota obtenido en las PNB-EM, también se indagó en las instituciones pertenecientes a las DRE de zonas fronterizas presentes en la clasificación de las primeras 100 instituciones a nivel nacional y el índice de desarrollo social (IDS, 2013) reportado por los cantones donde se encuentran ubicadas las instituciones educativas.

Las fuentes de información utilizadas para el estudio consistieron en las bases de datos de los resultados de las PNB-EM en el periodo 2013-2016, las cuales fueron proporcionadas por el Departamento de Estadística del MEP. También, se tomó en cuenta la información respecto a los indicadores sociales de los cantones a las que pertenecen las DRE del estudio consignado en el documento Índice de desarrollo social 2013, del MIDEPLAN.

\section{Procedimiento metodológico}

Los datos estadísticos con los que se trabajó fueron de dos ámbitos, el educativo en relación con las PNB-EM en lo que respecta a la promoción y nota promedio obtenida y el social relacionado con los valores de índice de desarrollo social cantonal del año 2013 (MIDEPLAN, 2013).

El índice de desarrollo social (IDS), como lo indica el MIDEPLAN (2013), utiliza cuatro dimensiones básicas y aporta un porcentaje al IDS, las cuales están relacionadas con la economía y corresponden a un peso del $30 \%$, la participación electoral con $18 \%$, salud aporta un $22 \%$ y la educación con 30\%; de ellas se despliegan los indicadores y sus respectivos índices, los cuales no se abordarán en detalle en esta investigación, pues están fuera del tema en discusión. Se resalta la clasificación del IDS, el cual va de mayor desarrollo entre un 100,0 a 72,5, posteriormente menor desarrollo que se divide de la siguiente manera: nivel medio 72,4 a 58,0; nivel bajo 57,9 a 43,9 y nivel muy bajo; 43,8 a 0,0. Con lo anterior se interpreta que el índice, cuanto más se acerca a uno presenta un alto porcentaje en relación con las dimensiones anteriormente señaladas y viceversa. 
doi: http://dx.doi.org/10.15359/ree.23-3.1

URL: http://www.una.ac.cr/educare

CORREO: educare@una.cr

En relación con el ámbito educativo, se utilizó la información disponible en los Informes Nacionales del Bachillerato de la Educación Formal de los años 2012, 2013, 2014, 2015 y 2016 con respecto a los porcentajes de promoción y nota promedio por cada una de las instituciones que aplicó dicha prueba en el período de estudio.

Particularmente, para el año 2016, ahondamos en el estudio de la lista de las primeras 100 posiciones, focalizando aquellas instituciones pertenecientes a las DRE que se toman en cuenta en el presente estudio.

\section{Análisis de los datos}

El análisis de las PNB-EM contempla lo territorial y toma en cuenta dos componentes: porcentaje de aprobación y promedio de nota, y su relación con el IDS, que a su vez se compone de cuatro indicadores (educación, economía, salud y participación). Para ello, se contextualizan e interpretan los resultados dentro de un espacio geográfico, en este caso las DRE en las zonas fronterizas norte y sur, con el fin de aportar a la política pública educativa. Para la interpretación de los datos se optó por gráficos, tablas de resumen de información y el apoyo de la cartografía como método que representa la facilidad de analizar territorialmente el dato.

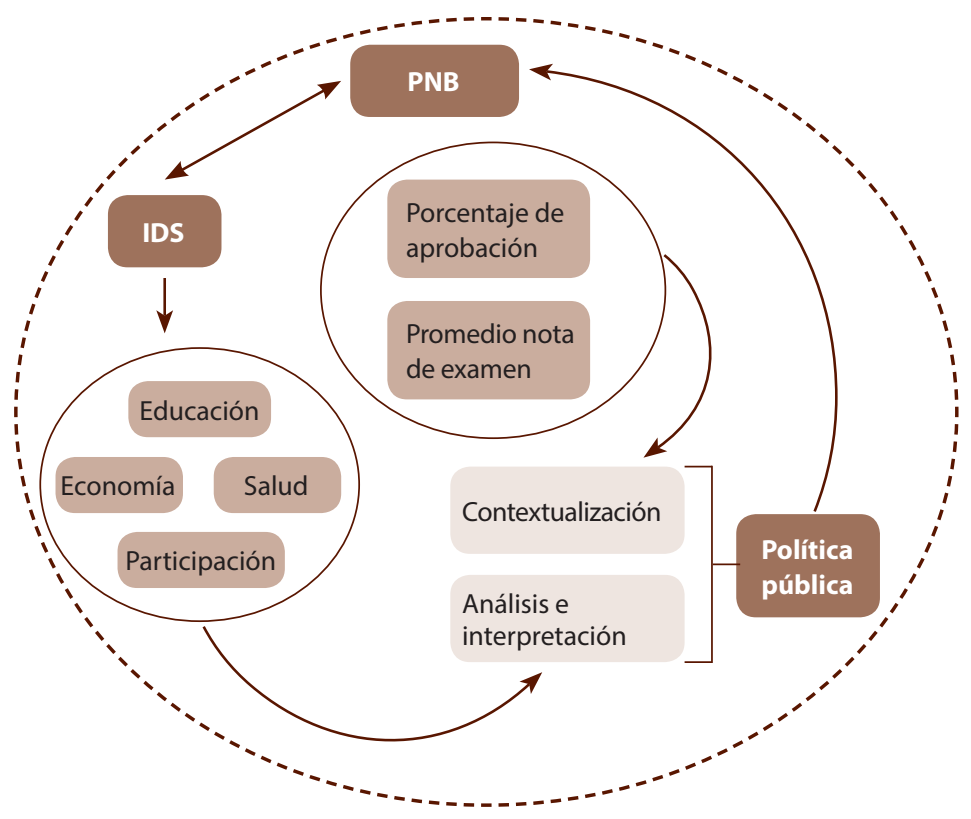

Figura 1: Elementos para la construcción territorial del análisis de datos de las PNB-EM.

Nota: Elaboración propia. 
En la Figura 1 se integra el análisis de dos categorías de las PNB-EM y su relación con el IDS cantonal desde la contextualización geográfica del estudio y su posible incidencia en las políticas públicas de educación.

\section{Categorías de análisis}

- Promoción y notas promedio en las pruebas nacionales de bachillerato en educación media en zonas fronterizas: analiza las variables "porcentaje de aprobación" y "promedio de nota" obtenida en las PNB-EM. La unidad de análisis es la institución educativa, agrupa los datos por DRE en el período 2013-2016.

- Instituciones pertenecientes a las DRE de zonas fronterizas presentes en la clasificación de las primeras 100 instituciones: analiza la participación de las instituciones pertenecientes a las DRE de zonas fronterizas en la lista que comprende los primeros 100 lugares de acuerdo con las variables "porcentaje de aprobación" y "promedio de nota" obtenida en las PNB-EM. La unidad de análisis es la institución educativa, de acuerdo con los datos reportados para el año 2016.

\section{Resultados, análisis y discusión}

El análisis de información se presenta en dos categorías, cada una muestra datos relevantes para el estudio con su respectiva descripción y caracterización de los resultados de las pruebas nacionales de bachillerato por región educativa fronteriza y en algunos casos la relación de estos datos con el IDS 2013.

Es importante aclarar que el MEP cuenta con un total de 27 DRE distribuidas en las 7 provincias del país, conforman las dependencias del Viceministerio Académico del MEP que organizan la prestación de servicios y facilitan la atención de las comunidades educativas.

Los datos analizados corresponden a los resultados que comprenden el periodo 2013 al 2016, toman en consideración las variables: porcentaje de aprobación y nota promedio obtenida en la prueba, además se adentra en el análisis de la clasificación de la lista de las primeras 100 instituciones de acuerdo con los dos parámetros mencionados anteriormente y en relación con su pertenencia a las DRE estudiadas. 
doi: http://dx.doi.org/10.15359/ree.23-3.1

URL: http://www.una.ac.cr/educare

CORREO: educare@una.cr

\section{Porcentaje de aprobación en las PNB-EM por región educativa}



Región educativa

Figura 2: Promedio de aprobación de las PNB-EM por DRE 2013-2016.

Nota: Elaboración propia con datos del MEP, Departamento de Análiis Estadístico (2017).

Según la Figura 2, en el periodo 2013-2016, se estimó un promedio nacional de 66 en la aprobación de las PNB-EM a nivel nacional, valor menor a 70 que corresponde a la calificación final mínima de aprobación para cada postulante de estas pruebas.

Durante ese mismo período de estudio, 12 de 27 DRE (Cartago, Occidente, Pérez Zeledón, Heredia, San José Oeste, San José Norte, Alajuela, San José Central, Turrialba, Desamparados, Los Santos y Guápiles) obtuvieron un porcentaje de aprobación en la PNB-EM mayor que el promedio nacional, y se encuentran en su mayoría dentro de la Gran Área Metropolitana (GAM). Al comparar los resultados, la DRE Cartago mostró el mayor rendimiento promedio a nivel nacional con un 79,6; es decir, la regional de Cartago superó en 13,6 unidades al promedio nacional.

En contraposición, 15 DRE (Puriscal, San Carlos, Coto, Cañas, Puntarenas, Grande de Térraba, Limón, Liberia, Santa Cruz, Aguirre, Nicoya, Peninsular, Sarapiquí, Zona Norte-Norte y Sulá) presentaron en ese mismo período un promedio inferior al nacional, lo cual muestra que más de la mitad de las DRE no superan la media nacional de PNB-EM. Llama la atención la DRE Sulá, DRE que contempla siete territorios indígenas (Telire, Talamanca Cabécar, Talamanca Bribri, Këkoldi, Tayní, Bajo Chirripó y Nayri Awayri) y que no solo fue la regional con menor promedio de aprobación $(45,3)$, sino que este valor se aleja alrededor de 20 unidades de la media de aprobación nacional, contexto que debe considerarse de cuidado y darle atención a dicha dirección. 
Asimismo, las DRE que obtuvieron un promedio menor de aprobación que el nacional se ubican, en su mayoría, fuera de la GAM en zonas rurales. Destacan, dentro de este grupo, aquellas que pertenecen a zonas costeras y fronterizas, en donde podría considerarse que estas áreas tienen menos recursos socioeconómicos y que, además, la concentración de la economía de Costa Rica se desarrolla dentro del Valle Central.

\section{Promedio de Nota en las PNB-EM por Región Educativa}

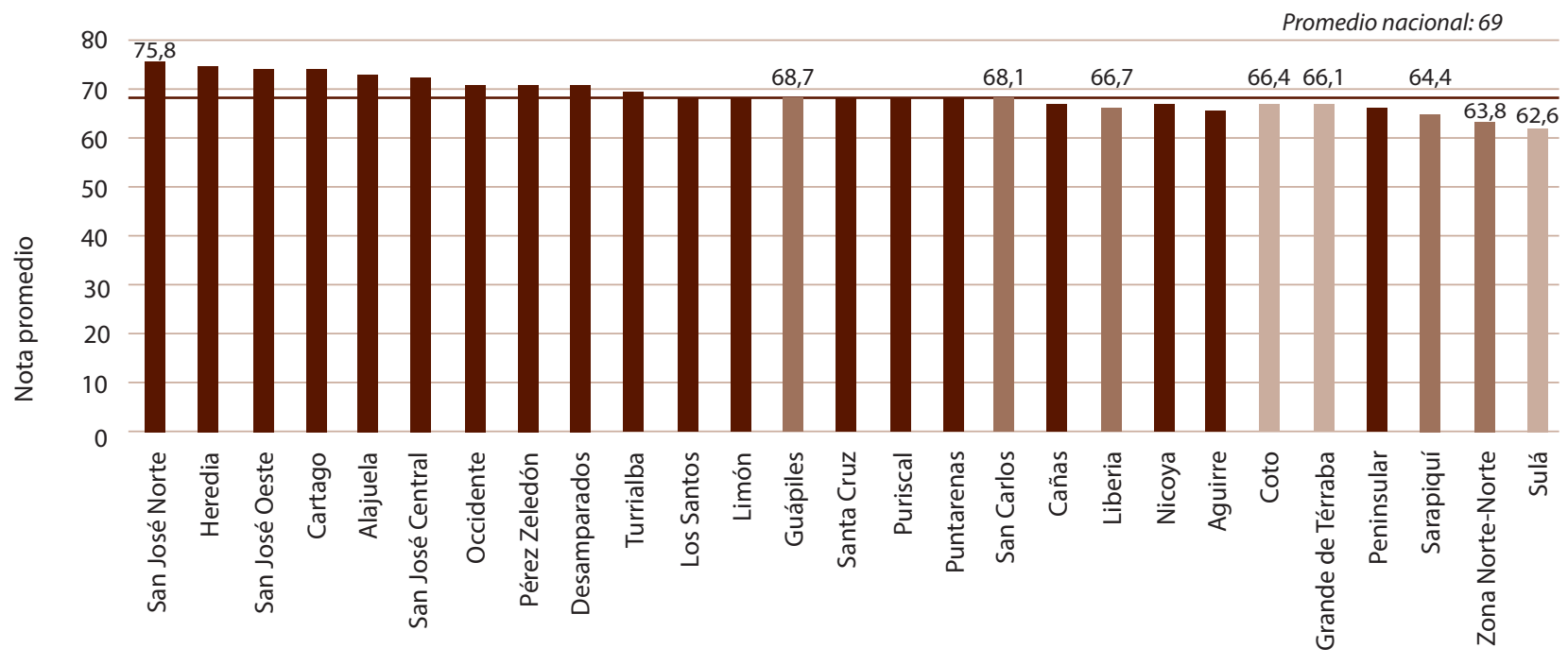

Región educativa

Figura 3: Nota promedio de las PNB-EM por DRE 2013-2016.

Nota: Elaboración propia con datos de MEP, Departamento de Análiis Estadístico (2017).

Según indica la Figura 3, el promedio nacional de nota en las PNB-EM del periodo 20132016 se calculó en 69, en una escala de 0 a 100. De las 27 DRE que componen el sistema educativo costarricense, la DRE San José Norte obtuvo la mayor nota promedio $(75,8)$ y la DRE de Sulá la menor nota promedio $(62,6)$ que además es inferior al promedio nacional.

De esas mismas 27 DRE, solo 11 superan el promedio nacional (San José Norte, Heredia, San José Oeste, Cartago, Alajuela, San José Central, Occidente, Pérez Zeledón, Desamparados, Turrialba y Los Santos), mientras que 16 (Limón, Guápiles, Santa Cruz, Puriscal, Puntarenas, San Carlos, Cañas, Liberia, Nicoya, Aguirre, Coto, Grande de Térraba, Peninsular, Sarapiquí, Zona Norte-Norte y Sulá) se encuentran por debajo del promedio nacional.

De la Figura 3, también se desprende que la totalidad de las DRE que se ubican en zonas fronterizas presentan un promedio de nota en la PNB-EM menor al promedio nacional. 
doi: http://dx.doi.org/10.15359/ree.23-3.1

URL: http://www.una.ac.cr/educare

CORREO: educare@una.cr

El análisis precedente demuestra que no corresponde a la misma DRE el mayor promedio de aprobación y el mayor promedio de nota, ya que estos fueron obtenidos por la DRE Cartago y San José Norte respectivamente. Situación contraria con la DRE Sulá que mostró el menor promedio de aprobación y de nota, por consiguiente, es necesario revisar las causas por las cuales en esta regional de educación fronteriza se obtienen resultados con tan bajo rendimiento en las PNB-EM.

\section{Resultados de las PNB según DRE fronterizas y desarrollo social}

El índice de desarrollo social (IDS) elaborado en el 2013 por MIDEPLAN refleja que las fronteras, según sus regiones educativas, cantón e índice son: para el caso del norte la Región Educativa Liberia compuesta por los cantones de La Cruz $(27,2)$, Liberia $(56,2)$, Bagaces $(42,2)$; Región Educativa Norte-Norte conformada por Guatuso $(23,5)$, Upala $(22,5)$ y Los Chiles $(14,9)$; Región Educativa San Carlos conformada por el cantón del mismo nombre $(40,6)$ al igual que la Región Educativa de Sarapiquí $(21,2)$; la Región Educativa Guápiles se basa territorialmente en el cantón de Pococí $(36.1)$ y Guácimo $(35,1)$.

Ahora bien, los datos parala frontera sur del país son similares a los expuestos anteriormente, por ejemplo: la Región Educativa Sulá a la cual le pertenece el cantón de Talamanca $(0,0)$; Región Educativa Grande de Térraba con el cantón de Buenos Aires $(16,1)$ y Osa $(29,2)$; por último la Región Educativa de Coto con los cantones de Coto Brus $(28,3)$, Golfito $(21,4)$ y Corredores $(25,1)$.

Es de resaltar que los datos anteriormente señalados reflejan que los cantones que conforman las regiones educativas fronterizas, tanto del sur del país como al norte, presentan un IDS muy bajo, a excepción del cantón de Liberia con un índice bajo. Se interpreta que, tal y como se mencionó en la metodología del presente documento, al presentar un índice bajo o muy bajo el peso de las dimensiones presenta porcentajes también bajos, lo que indica que las áreas en estudio presenta deficiencias en economía, participación electoral, salud y educación.

El caso del cantón de Liberia es notorio, debido a que es el único que supera un índice del 50 , pues en promedio los 16 cantones llegan apenas a una cuarta parte $(27,4)$ del índice total. Estas regiones educativas, junto con sus cantones, posee espacialmente la connotación de ser fronteras vistas históricamente como áreas rezagadas, y la percepción de la población, tal como lo señala Meza (2008), son vistas como espacios "... generalmente marginados y sujetos de apoyos especiales por parte del Estado" (p. 20). 




Figura 4: DRE fronterizas según IDS reportado.

Nota: Imagen de archivo de esta investigación, elaborado con datos de MIDEPLAN (2013).

A su vez, los cantones que pertenecen a estas DRE ocupan los últimos trece puestos en el IDS cantonal del 2013. En consecuencia, podemos observar una estrecha relación entre los indicadores de las condiciones socioeconómicas de las DRE que se encuentran en zonas fronterizas y los bajos resultados reportados en las PNB-EM; cabe destacar que todas las DRE del estudio se encuentran en los índice más bajo del IDS, como se puede observar en la Figura 4. Además, es claro que, conforme se aleja del centro del país hacia la periferia, la desigualdad va en aumento y que la posibilidad de acceder y disfrutar de derechos básicos, como señala en el IDS 2013, son cada vez menores, tanto en cuanto a tener un ingreso suficiente con la finalidad de una vida digna; como en la participación electoral, donde la cohesión social es menor; en la salud los niveles de vida sana y saludable van en detrimento y, no menos importante, la educativa asociada a la disponibilidad y el acceso a esta; ello evidencia que el acceso a derechos que podrían considerarse tan básicos podrían estar en cuestionamiento. Es claro que la GAM concentra más del $60 \%$ de la población total de Costa Rica, pero no hay que olvidar que mucha de la economía costarricense proviene de las regiones aquí analizadas, tanto del sector turismo como del agrario, por citar algunas. 
doi: http://dx.doi.org/10.15359/ree.23-3.1

URL: http://www.una.ac.cr/educare

CORREO: educare@una.cr

A partir de los resultados expuestos en las PNB-EM en las zonas fronterizas y tomando en consideración que el colectivo estudiantil que obtiene dichos resultados proviene de zonas geográficas con un IDS "muy bajo", es importante destacar que este índice contempla indicadores económicos, de participación social, salud y educación; en cuanto a educación se toman en consideración indicadores como: las escuelas sin servicio eléctrico, agua potable, aulas en mal estado, escuelas unidocentes, porcentajes de matrícula y reprobación. Esto nos lleva a plantear qué características de exclusión social, en una zona geográfica determinada, son un problema incidente en la cultura y, por ende, en la educación en general, tal como lo menciona Kaplan (2006) en el sentido de que el éxito o fracaso educativo constituye una construcción cultural.

Apegados al planteamiento de Bourdieu (1998), la desigualdad en las condiciones sociales y simbólicas de acceso, permanencia y aprobación escolar intervienen en los procesos diferenciados a nivel educativo; de esta forma, el capital cultural es desigualmente "acumulado" por los sujetos. Y, por ende, el sistema educativo legitima la cultura que corresponde a la clase social dominante, generando diferencias de forma implícita entre el estudiantado y normalizando las diferencias sociales como herencias naturales.

A partir de la perspectiva de Bourdieu (1998), se puede considerar que en una misma zona geográfica, pero en condiciones educativas diferentes, es posible generar resultados disímiles, como sucede con los resultados obtenidos en las PNB-EM en zonas fronterizas de colegios de modalidad científica, humanista y colegios de condición legal privada en comparación con las instituciones educativas de modalidad pública. En consecuencia, para Kaplan (2006, p. 35) "no se puede interpretar las desigualdades y diferencias socioculturales de los estudiantes por fuera de las formas sociales de valoración y rechazo de ciertos individuos y grupos".

\section{Rendimiento de colegios en DRE fronterizas en el 2016}

Tal como se analizó en el periodo 2013-2016, las DRE del estudio nos mostraron valores de aprobación en las PNB-EM que superen en mayor medida al promedio nacional. Por ello interesa profundizar aún más en los datos que presentan los colegios que conforman las regiones de educación del estudio.

Las 8 DRE pertenecientes a zonas fronterizas norte y sur, para el 2016 registran un total de 305 colegios de las distintas modalidades de educación secundaria existentes, de este total 287 corresponden a colegios de condición legal pública, 17 a centros educativos privados y 1 de condición subvencionada.

En el 2016, el promedio nacional de nota en PNB-EM fue 80,46. Cabe señalar que de los colegios analizados, el $43 \%$ muestra un porcentaje de promoción en las PNB-EM superior a 70, el cual representa 133 colegios y de estos un predominio de condición legal pública (85\%). En consecuencia, menos de la mitad de los colegios de las zonas fronterizas norte y sur presentan una promoción superior al mínimo establecido representados en su mayoría como colegios públicos. 
En adición, solo 19 de 305 colegios logran un promedio de nota de prueba nacional de bachillerato superior a 90, por lo que se puede distinguir el escaso número de instituciones que podrían clasificarse "de excelencia" tomando en cuenta el rendimiento en estas pruebas. De los 19 colegios, 12 corresponden a centros privados y 7 a colegios públicos de modalidad científica, humanista, liceo rural y telesecundaria, lo que indica que en estas regiones la condición "colegio de excelencia" es representado en su mayoría por colegios privados y por colegios académicos que por su modalidad se enfocan a la profundización y estudios complementarios de las asignaturas del currículo nacional básico para educación diversificada. Asimismo, el $94 \%$ de las instituciones de secundaria de las zonas fronterizas no lograron obtener rendimientos de excelencia.

En las regiones de educación Coto, Sarapiquí, San Carlos y Guápiles el porcentaje de promoción fue aproximadamente un 50\%, mientras que en las regiones Grande de Térraba y Zona Norte-Norte la promoción en estas pruebas fue menor al 65\%, zonas en desventaja social que albergan respectivamente cantidad importante de población indígena y migrantes. En condición alarmante se encuentra la DRE Sulá con un 75\% de no aprobación en las PNB-EM.

\section{Participación de las DRE fronterizas en la clasificación de los 100 primeros colegios del año 2016}

Tabla 2: Colegios pertenecientes a las DRE fronterizas en los primeros 100 lugares según clasificación del año 2016

\begin{tabular}{|c|c|c|c|c|c|}
\hline Posición en la lista & Nombre de la institución & Tipo & Región & Cantón & IDS \\
\hline 4 & Científico San Carlos & Pub & San Carlos & San Carlos & 40,60 \\
\hline 8 & Científico Liberia & Pub & Liberia & Liberia & 56,20 \\
\hline 16 & Pindeco & Pri & Grande Térraba & Buenos Aires & 16,10 \\
\hline 32 & Valle del Sol & Pri & Guápiles & Pococí & 36,10 \\
\hline 36 & Humanístico de Coto & Pub & Coto & Corredores & 25,10 \\
\hline 48 & Green Forest School & Pri & San Carlos & San Carlos & 40,60 \\
\hline 54 & Liceo Rural Changuena & Pub & Grande Térraba & Buenos Aires & 16,10 \\
\hline 56 & John F. Kennedy High School & Pri & Coto & Coto Brus & 29,30 \\
\hline 62 & Escuelas Internacionales Cristianas & Pri & Liberia & Liberia & 56,20 \\
\hline 75 & Sistema Educativo Cenit & Pri & Liberia & Liberia & 56,20 \\
\hline 76 & San Diego Earth & Pri & Guápiles & Guácimo & 35,10 \\
\hline 80 & Colegio María Inmaculada & Sub & San Carlos & San Carlos & 40,60 \\
\hline 86 & T. V. el Castillo fortuna & Pub & San Carlos & San Carlos & 40,60 \\
\hline 87 & Bil. San Francisco de Asís & Pri & Guápiles & Pococí & 36,10 \\
\hline
\end{tabular}

Nota: Elaboración propia con datos de MEP, DGEC (2017) y MIDEPLAN (2013). 
doi: http://dx.doi.org/10.15359/ree.23-3.1

URL: http://www.una.ac.cr/educare

CORREO: educare@una.cr

Con base en el promedio de nota obtenido en las PNB-EM y el porcentaje de aprobación, en el 2016 se crea una clasificación a nivel de instituciones en el territorio nacional, tan solo 14 colegios pertenecen a alguna de las regiones de educación del estudio, de ahí que solo esta cantidad aparece en la lista y logran ocupar un puesto entre los lugares 4-87 en dicha clasificación. Además, con excepción de los 5 colegios de la región de Liberia, los otros 9 de los 14 que se señalan en la Tabla 2, todos ellos pertenecen a cantones con IDS muy bajo, lo cual refleja que en las regiones fronterizas se acentúan condiciones de desigualdad que se reflejan en el sistema educativo.

De estos 14 colegios de regionales educativas fronterizas, solo el Colegio Científico de San Carlos (posición 4) y el Colegio Científico de Liberia (posición 8) están dentro de los primeros diez mejores puestos de aprobación en las PNB-EM, tal como lo muestra la Tabla 2.

En esa misma línea, destaca el colegio Pindeco de condición legal privada que pertenece al cantón de Buenos Aires y que ocupa el puesto 16 dentro de los primeros 100, así como el Liceo Rural Changuena de condición legal pública que ocupa la posición 54 , dado que ambos colegios se encuentran en un cantón con IDS muy bajo $(16,10)$, lo cual refleja algunas de las asimetrías en los rendimientos de aprobación de centros educativos, pero que no reflejan distinción para este caso particular de los contextos geográficos. Es decir, si bien es cierto el cantón de Buenos Aires en su contexto de desarrollo territorial es claramente desigual en contraste con el cantón de Escazú, en tema de condición legal, el colegio anteriormente señalado llega a equiparse con muchos otros colegios que están ubicados en la GAM.

De los 14 colegios que pertenecen a zonas fronterizas que forman parte de la clasificación de los primeros 100 lugares, 8 son de tipo privado, 1 es subvencionado y 5 son públicos; en consecuencia, la condición legal privada de estos colegios predomina con mejores resultados en las PNB-EM en las zonas geográficas fronterizas.

Llama la atención que de los 5 colegios públicos que ocupan un puesto en esta clasificación, 3 son de corte científico o humanista, únicamente uno es de modalidad liceo rural (Liceo Rural Changuena) ubicado en la DRE Grande de Térraba en el cantón de Buenos Aires y uno de Telesecundaria (T.V El Castillo Fortuna) perteneciente a la DRE de San Carlos del cantón de San Carlos. Lo anterior indica que otros colegios de las más de 22 modalidades de educación secundaria del sistema educativo costarricense no ocupan una posición en la clasificación de los primeros 100 colegios por su rendimiento de aprobación. Esta condición la cumplen las DRE Sarapiquí, Zona Norte-Norte (ambas de la frontera norte) y Sulá (frontera sur) que no logran incluir ninguna de sus instituciones en la lista de los primeros 100 colegios. 


\section{Conclusiones}

Dentro de las conclusiones más relevantes podemos destacar que las 8 DRE del presente estudio tienen un rendimiento inferior al promedio nacional en pruebas de bachillerato.

Los cantones que conforman cada una de las DRE del estudio ocupan los puestos más bajos en el IDS cantonal 2013. Esto confirma una estrecha relación entre los bajos resultados de las PNB-EM y los cantones con índices de desarrollo bajo en zonas fronterizas. Esta relación entre PNB-EM, IDS y contextualización de zonas fronterizas es una triada que genera resultados que desfavorecen al estudiantado evaluado mediante las PNB-EM en contextos de alta vulnerabilidad y con población históricamente rezagada.

En la lista de los primeros 100 colegios de los resultados de las PNB-EM, no aparecen colegios de las direcciones regionales de educación Sarapiquí, Zona Norte-Norte (ambas de la frontera norte) y Sulá (frontera sur); las otras direcciones regionales cuentan con una participación mínima y corresponden a colegios de corte científico o humanista, un liceo rural y una telesecundaria. Ello permite visualizar algunas modalidades y tipos de instituciones que se encuentran en desventaja como los colegios académicos de jornada diurna que son la mayoría y otros como los de jornada nocturna.

Es importante valorar los resultados de las PNB-EM a la luz de las realidades sociales y culturales, tomando en consideración el índice de desarrollo social y el contexto en el que se encuentran el colectivo estudiantil. Estos resultados nos permiten abrir espacios de reflexión, análisis y diálogo sobre los mismos resultados de las PNB-EM, sus repercusiones en la población en general y, sobre todo, en aquellas poblaciones que se encuentran en condiciones de mayor vulnerabilidad social.

Las DRE fronterizas presentan grandes deficiencias en términos de IDS, por lo que resulta oportuno focalizar políticas públicas para dichas áreas relacionadas con temas económicos, participación electoral, saludy, claramente, educación, sin perder de vista que las áreas fronterizas poseen una connotación diferenciada al resto del país, por su influencia transfronteriza.

\section{Referencias}

Barrantes, F. (3 de noviembre de 1997). La regionalización del sistema educativo costarricense. Mito y realidad. Coloquio Regional sobre Descentralización de la Educación en América Central, Cuba y República Dominicana. Coloquio llevado a cabo en San José, Costa Rica: CLAD. Recuperado de http://old.clad.org/documentos/otros-documentos/la-regionalizaciondel-sistema-educativo-costarricense.-mito-y-realidad 
doi: http://dx.doi.org/10.15359/ree.23-3.1

URL: http://www.una.ac.cr/educare

CORREO: educare@una.cr

Bourdieu, P. (1998). La distinción. Criterios y bases sociales del gusto. (Trad. María del Carmen Ruiz de Elvira). España: Grupo Santillana de Ediciones S. A. Recuperado de http://ceiphistorica. com/wp-content/uploads/2016/01/Bourdieu-Pierre-La-distinci\%C3\%B3n.pdf

Calderón, L. G. (2015). Costa Rica: Um estudo geográfico da pobreza e a contribuição das políticas públicas (tesis de maestria). Universidade Federal do Rio Grande.

Castillo, S. (2002). Compromisos de la evaluación educativa. Madrid: Pearson educación.

Esquivel, J., Montero, E., Sosa, Doris., Hernández, A., Corella, M. y Fallas, J. (2006). Evaluación externa de las pruebas nacionales de bachillerato de la educación media, Informe final. San Pedro, Costa Rica: Consejo Superior de Educación.

Hernández, R., Fernández C. y Baptista, P. (2014). Metodología de la investigación (5ª ed.). México: McGraw-Hill.

Kaplan, C. V. (2006). La inclusión como posibilidad. Buenos Aires: Ministerio de Educación., Ciencia y Tecnología de la Nación.

López, N. (2005). Equidad educativa y desigualdad social: Desafíos de la educación en el nuevo escenario latinoamericano. Buenos Aires: IIEP- UNESCO. Recuperado de http://unesdoc. unesco.org/images/0014/001425/142599s.pdf

Meza, N. (2008). Espacios regionales fronterizos. Teoría, política y práctica del desarrollo y la integración fronteriza. Recuperado de http://www.eumed.net/libros-gratis/2008b/400/ index.htm\#indice

Ministerio de Educación Pública. (6 de febrero de 2018). Reglamento de evaluación de los aprendizajes 2018, DecretoN. ${ }^{\circ}$ 40862-MEP.LaGacetaDiarioOficial.AlcanceN. ${ }^{\circ 26 .}$. Recuperado de https://www.imprentanacional.go.cr/pub/2018/02/06/ALCA26 0602 2018.pdf

Ministerio de Educación Pública, Departamento de Análisis Estadístico. (2017). Resultados de las Pruebas Nacionales de Bachillerato de Educación Media 2013-2016 [Archivo de datos]. San José, Costa Rica: Autor.

Ministerio de Educación Pública, Dirección de Gestión y Evaluación de la calidad. (2017). Informe nacional. Bachillerato de la educación formal 2016. Rendimiento y niveles de desempeño. San José, Costa Rica: Autor. Recuperado de http://www.dgec.mep.go.cr/sites/all/files/ dgec mep go cr/documentos/informe nacional 2016 0.pdf

Ministerio de Planificación Nacional y Política Económica (MIDEPLAN). (2013). Índice de desarrollo social 2013. Recuperado de https://www.tec.ac.cr/sites/default/files/media/doc/indice desarrollo social 2013 resumen $0 . p d f$ 
doi: http://dx.doi.org/10.15359/ree.23-3.1

URL: http://www.una.ac.cr/educare

Pérez, R. (2015). La competencia digital en un programa para jóvenes desaparecidos que han desertado de la secundaria (Tesis doctoral). Universidad Autónoma de Madrid. Recuperado de $\quad$ http://repositorio.uam.es/bitstream/handle/10486/669591/perez aguirre rosina. pdf? sequence $=1$

Programa Estado de la Nación. (2013). Cuarto informe estado de la educación. San José, Costa Rica: Autor. Recuperado de https://estadonacion.or.cr/informe-iv-estado-educacion

Stubbs, S. (2008). Inclusive education: Where there are few resources ( $2^{\mathrm{a}}$ ed.). Oslo, Norway: The Atlas Alliance. Recuperado de https://www.eenet.org.uk/resources/docs/IE\%20few\%20 resources\%202008.pdf

UNESCO. (2012). Lucha contra la exclusión en la educación. Guía de evaluación de los sistemas educativos rumbo a sociedades más inclusivas y justas. París: Autor. Recuperado de http:// unesdoc.unesco.org/images/0021/002170/217073s.pdf 